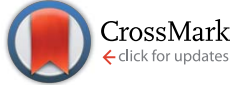

Cite this: J. Mater. Chem. A, 2015, 3, 15745

Received 19th May 2015

Accepted 12th June 2015

DOI: $10.1039 / c 5 t a 03646 a$

www.rsc.org/MaterialsA

\section{Cellulose nanofibril core-shell silica coatings and their conversion into thermally stable nanotube aerogels $\uparrow$}

\author{
Dongming Liu, ${ }^{a}$ Qiong Wu, ${ }^{a}$ Richard L. Andersson, ${ }^{a}$ Mikael S. Hedenqvist, ${ }^{a}$ \\ Stefano Farris ${ }^{\mathrm{b}}$ and Richard T. Olsson*ac
}

\begin{abstract}
A facile water-based one-pot reaction protocol for obtaining $20 \mathrm{~nm}$ thick uniform silica coatings on cellulose nanofibrils (CNFs) is herein presented for the first time. The fully covering silica shells result in the thermal stability of the CNFs improved by $\mathrm{ca} .70{ }^{\circ} \mathrm{C}$ and $50{ }^{\circ} \mathrm{C}$ under nitrogen and oxygen atmospheres, respectively. Heating of the core-shell hybrid fibres to $400{ }^{\circ} \mathrm{C}$ results in complete degradation/removal of the CNF cores, and demonstrates an inexpensive route to large-scale preparation of silica nanotubes with the CNFs used as templates. The key to a uniform condensation of silica (from tetraethyl orthosilicate) to cellulose is a reaction medium that permits in situ nucleation and growth of the silica phase on the fibrils, while simultaneously matching the quantity of the condensed silica with the specific surface area of the CNFs. Most coatings were applied to bundles of 2-3 associated CNFs, which could be discerned from their negative imprint that remained inside the silica nanotubes. Finally, it is demonstrated that the coated nanofibrils can be freeze-dried into highly porous silica/cellulose aerogels with a density of $0.005 \mathrm{~g} \mathrm{~cm}^{-3}$ and how these hybrid aerogels preserve their shape when extensively exposed to $400{ }^{\circ} \mathrm{C}$ in air (>6 h). The resulting material is the first reported silica nanotube aerogel obtained by using cellulose nanofibrils as templates.
\end{abstract}

\section{Introduction}

Thermally resistant cellulose nanofibrils (CNFs) are of interest in many envisioned applications where the cellulose "building block" may be exposed to temperatures different from its natural environments. From an engineering perspective, thermally stable CNFs could be used as fillers for reinforcement in polymers processed at higher temperatures, but also in the development of porous and more heat-resistant bio-based insulation materials. ${ }^{1-6}$ Other applications may include gas- and liquid-barrier agents in packing materials, or as paper additives. $^{{ }^{1-6}}$ However, the cellulose structure decomposes into carbon residues by the oxidation of the $\beta(1 \rightarrow 4)$-glycosidic ether bonds in the cellulose backbone (depolymerisation,

${ }^{a}$ Department of Fibre and Polymer Technology, School of Chemical Science and Engineering, KTH Royal Institute of Technology, Stockholm, Sweden. E-mail: rols@ kth.se

${ }^{b}$ DeFENS, Department of Food, Environmental and Nutritional Sciences-Packaging Division, University of Milan, via Celoria 2, 20133 Milan, Italy

${ }^{c}$ Wallenberg Wood Science Center (WWSC), KTH Royal Institute of Technology, SE-100 44 Stockholm, Sweden

$\dagger$ Electronic supplementary information (ESI) available: Video portraying the formation of silica nanotube aerogels on the degradation of the cellulose nanofibril template and the X-ray diffraction pattern showing the elimination of the cellulose fibril support used to prepare the silica nanotube aerogels. See DOI: $10.1039 / \mathrm{c} 5$ ta03646a reduction of DP) at $c a .220^{\circ} \mathrm{C}$, followed by decomposition of the cyclic carbon glucose residues into char and gas $\left(>300{ }^{\circ} \mathrm{C}\right) \cdot{ }^{7-10}$ This degradation is catalytically enhanced (resulting in premature degradation) by sulphite groups remaining on the surface of the CNFs from the sulphuric acid extraction used to isolate the fibrils from their natural habitat, or by the presence of metal/metal oxide nanoparticles, e.g. silver, gold, platinum, titanium, zirconium and aluminium..$^{11-15}$

In the past, flame-retardant finishes containing nitrogen and phosphorus compounds have been reported for cotton cellulosic fabrics. ${ }^{16-18}$ These coatings provide a temporarily lower susceptibility of the cellulose to undergo thermal degradation but the effect is inevitably limited by their quantities and consumption rates. ${ }^{\mathbf{1 9 , 2 0}}$ The use of alternative acids during the CNF extraction procedure provides a first step towards preventing the thermal degradation of the CNFs, e.g. hydrochloric or phosphoric acid. ${ }^{21}$ Espinosa et al. recently compared phosphoric acid with sulphuric acid and showed that phosphoric acid induced substantially less catalytic action during the degradation of CNFs at elevated temperatures. ${ }^{22}$ However, the influence of the different acid remains on the degradation mechanisms is unclear in terms of initiation, oxidation and/or influence of mechanistic degradation patterns (char formation), and in many cases the thermal stability of the raw (untreated) cellulose material prevails over that of the extracted CNF material. ${ }^{23-26}$ 
Heat-resistant coatings that improve the thermal performance of virgin cellulose nanofibrils have received significantly less attention than the selection of the acid used for the isolation of the CNFs. Generic CNF surface coating-modification methods to raise the onset temperature of cellulose degradation are therefore missing. To some extent this can be related to the extraction and processing of cellulose nanofibrils with characteristics affected by the adopted conditions and possible impurities, but also due to the fact that cellulose nanofibrils disclose different properties depending on the origin of the CNF crystals. Some of these differences in CNF characteristics were recently described by Sacui et al. in terms of size, morphology, crystallinity, surface energy, and chemical character. ${ }^{27}$ This inevitably affects the possibilities to develop generic methods for improved thermal performance of the CNFs, e.g. since most surface modifications ultimately rely on predictable surfaces to be modified.

In this article, we demonstrate how it is possible to improve the thermal performance of CNFs extracted from bacterial cellulose by encapsulating the individual fibrils in uniform layers of silica. The necessary reaction conditions for preparing these silica-cellulose hybrid fibres are presented for the first time, opening the avenue for large-scale preparation of more heat-resistant "core-shell" cellulose/silica hybrid nanofibrils. The thermal performance was improved by $c a .70{ }^{\circ} \mathrm{C}$ in nitrogen and $50{ }^{\circ} \mathrm{C}$ in oxygen. The silica-coated CNFs were further heated to $>400{ }^{\circ} \mathrm{C}$ to remove the cellulose cores, which resulted in silica nanotubes with internal dimensions equivalent to the dimensions of the original cellulose nanofibrils. Previously, research has been focused on the preparation of silica nanotubes by deposition on porous aluminium oxide substrates, ${ }^{28}$ coating of carbon nanotubes, ${ }^{29}$ organic molecular deposition (including surfactants, $^{30}$ acids, ${ }^{31}$ block copolymers ${ }^{32}$ and gels $\left.^{33,34}\right)$, or coating and etching of $\mathrm{TiO}_{2} / \mathrm{CTAB}$ pre-treated cotton fibers ${ }^{35}$ as templates. ${ }^{36}$ It is herein demonstrated that cellulose may present a viable alternative for a large-scale and more economic preparation of several micrometres long silica nanotubes via the presented templating reactions. The coated CNFs were also freeze-dried into porous cellulose/silica aerogels that were heated at $400{ }^{\circ} \mathrm{C}$ for 6 hours in air, thereby demonstrating a method to transform the "core-shell" nanofibers to the first reported silica nanotube aerogels.

\section{Experimental section}

\subsection{Preparation of cellulose mats from bacteria cultures}

Bacterial cellulose was grown from a 20 litre sterilized glucose solution inoculated with Acetobacter xylinum - ATCC 23767. ${ }^{27,37}$ The solution composition was per litre: $20 \mathrm{~g}$ of D-glucose (dextrose, ACS reagent, Sigma-Aldrich), $5 \mathrm{~g}$ of yeast extract (Fluka, CAS 8013-01-2), $1.15 \mathrm{~g}$ of anhydrous citric acid (reagent grade, Scharlau), $5.7 \mathrm{~g}$ of magnesium sulphate (puriss, p.a., Fluka), $12.25 \mathrm{~g}$ of triphosphate water from Scharlau $(5.0 \mathrm{~g}$ of peptone, $2.5 \mathrm{~g}$ of sodium chloride, $4.5 \mathrm{~g}$ of disodium phosphate, and $0.75 \mathrm{~g}$ of potassium phosphate), and 1 vol\% ethanol (96\%). The $20 \mathrm{~L}$ solution was inoculated with a $150 \mathrm{~mL}$ pre-inoculated and fully developed bacteria culture that had been scaled up from a $5 \mathrm{~mL}$ sample prepared from the preserved strain in the glass ampoule. The time required to generate the fully developed bacteria culture and uniformly growing cellulose pellicle mats in each pre-inoculated sample was $48 \mathrm{~h}$ at $28{ }^{\circ} \mathrm{C}$. The weight of the wet microbial cellulose pellicle mat formed on the surface of the scaled up growth medium after another 48 hours was $c a .1 .0 \mathrm{~kg}$ (including the growth medium inside the mat). The mats were cut into $1 \mathrm{~cm}^{3}$ cubes that were boiled repeatedly in distilled water $(5 \times)$ until no residual growth medium could be detected. The pieces were then boiled twice in $10 \mathrm{vol} \%$ concentrated $\mathrm{NaOH}(2 \mathrm{~L} \times 2)$ for $20 \mathrm{~min}$, with intermediate rinsing with distilled water until neutral $\mathrm{pH} \approx 7$.

\subsection{Extraction of cellulose nanofibrils by acid hydrolysis}

Prior to the extraction of the CNFs, the $1.0 \mathrm{~kg}$ of bacterial cellulose cubes was shredded using a regular kitchen blender and compressed using a SEFAR polyamide mesh (PA 1000 120/ $305-35 \mathrm{~W}$ with $46 \mu \mathrm{m}$ openings) to remove most of the water. This was performed to facilitate spreading/distribution and acid diffusion into the cellulose material during the early stages of the hydrolysis reaction, i.e. for a more even degradation of the amorphous parts. The total mass of wet cellulose was $148 \mathrm{~g}$. The water remaining in the compressed cellulose was $87.2 \pm 0.8 \%$ (evaluated from 3 samples), corresponding to the dry bacterial cellulose content of 18.9 g. Fig. S1 $\uparrow$ shows the bacterial cellulose during the removal of growth medium (a) and the shredded material before exposure to acid (b).

$148 \mathrm{~g}$ of wet cellulose was added to a $2 \mathrm{~L}$ aqueous hydrochloric acid solution containing $30 \mathrm{vol} \%$ of reagent grade acid (Merck KGaA), and stirred at $60 \pm 0.5{ }^{\circ} \mathrm{C}(300 \mathrm{rpm})$. The suspension was stirred for 9 hours until no visible cellulose pieces were apparent and the solution had acquired an even beige colour. The extraction was terminated by the addition of 2 L cold MilliQ water and isothermally centrifuged at $10000 \mathrm{rpm}$ $\left(15{ }^{\circ} \mathrm{C}\right)$ for $10 \mathrm{~min}$, four times. Between the centrifugation cycles, the cellulose was collected at the bottom of the centrifuge tubes; the acidic supernatant was decanted and replaced with fresh MilliQ water $\left(50{ }^{\circ} \mathrm{C}\right)$, followed by redispersion using a high-shear mixer (Ultra Turrax DI-25) for $5 \mathrm{~min}$. After the third cycle, a neutral $\mathrm{pH}$ was obtained and the supernatant was transparent. By the last addition of MilliQ water, the sample was adjusted to a $0.4 \mathrm{wt} \%$ solid content in the pure water. The total amount of extracted CNFs was $10.4 \mathrm{~g}$ ( $55 \mathrm{wt} \%$ yield), from $148 \mathrm{~g}$ of shredded cellulose (or $18.9 \mathrm{~g}$ of corresponding dry bacterial cellulose). In all subsequent experiments, this cellulose containing $4 \mathrm{mg}$ of pure CNFs per $\mathrm{mL}$ was used. The specific surface area of the CNFs was calculated to be $c a .159 \mathrm{~m}^{2} \mathrm{~g}^{-1}$ from size distributions obtained by manual counting of a minimum of 500 fibrils deposited on TEM grids, which is in agreement with the calculated value of $189 \mathrm{~m}^{2} \mathrm{~g}^{-1}$ by Roman et al. ${ }^{14}$ and a lower experimental value of $103 \mathrm{~m}^{2} \mathrm{~g}^{-1}$ for dry bacterial cellulose fibrils by Olsson et al. ${ }^{38}$

\subsection{Silica coating of cellulose nanofibrils}

Tetraethyl orthosilicate (TEOS, $\geq 98 \%$ ) and ammonium hydroxide (aq. 28 wt\%) were purchased from Sigma-Aldrich. 
Methanol, ethanol and 2-propanol were obtained from VWR (reagent grade, $\geq 99.8 \%$ ). High-resistivity MilliQ water ("Type 1", following ISO 3696 and ASTM D1193-91, defined as 18.2 Mohm $\mathrm{cm}$ at $25{ }^{\circ} \mathrm{C}$ ) was used in all the sample preparation procedures. The methanol, ethanol or 2-propanol reaction media, which contained 18 vol\% water, was used due to the different abilities of these alcohols to slow down the silica precursor condensation reaction. ${ }^{39}$ The evaluated reaction media were methanol, ethanol or 2-propanol, which contained 18 vol\% water. Ammonia was used due to its previously reported ability to catalyse the Stöber reaction. ${ }^{40}$ In all reactions, the addition of the concentrated ( $\geq$ 98\%) tetraethyl orthosilicate (TEOS) was made as a final step to the reaction suspension to allow the TEOS to hydrolyse and initiate its condensation in situ onto the CNFs, i.e. to avoid premature self-nucleation of the silica precursor into solitary silica particles that occurs if the TEOS would have been given time to hydrolyse in absence of the CNFs. ${ }^{40}$

\subsection{Determination of the cellulose to TEOS ratio and effect of alcohols on condensation chemistry}

A sample of $0.67 \mathrm{~mL}$ (2.7 $\mathrm{mg}$ CNFs) or $2.0 \mathrm{~mL}$ ( $8 \mathrm{mg}$ CNFs) from the extracted suspension was centrifuged, decanted from water and redispersed thrice via solvent exchange into a $2.55 \mathrm{~mL}$ mixture of $82 \mathrm{vol} \%$ alcohol (methanol, ethanol or 2-propanol) and MilliQ water in a $50 \mathrm{~mL}$ polypropylene tube. The CNF suspension was then ultrasonicated in an ultrasonic bath for 5 min at an average intensity of $300 \mathrm{~W}$. The polypropylene tube (reactor) was then mounted onto a vortex shaker (Vortex Genie 1, USA) and $0.057 \mathrm{~mL}$ aqueous ammonia solution was injected into the reactor. Finally, the coating condensation reaction was initiated by adding $0.06 \mathrm{~mL}$ tetraethyl orthosilicate (TEOS). The amount of TEOS used was calculated to yield a complete coverage of fully condensed silica on the CNFs, and the ratio of aqueous ammonia to TEOS was kept constant for all reactions. The reaction proceeded at $20{ }^{\circ} \mathrm{C}$ (under maximum shaking) for $3 \mathrm{~h}$ to achieve complete condensation of the silica phase. The resulting core-shell CNFs were centrifuged for 90 seconds at 1000 RCF in a tabletop Eppendorf Minispin centrifuge and washed three times with ethanol. The most favourable reaction medium was established using $0.67 \mathrm{~mL}$ of the extracted CNF suspension $(2.7 \mathrm{mg}$ ). After mixing all the components, the masses of cellulose in the reaction tube were $1 \mathrm{mg} \mathrm{mL}^{-1}$ or $3 \mathrm{mg}$ $\mathrm{mL}^{-1}$. All samples are referred to as the mass of fibrils modified in the reaction tube per volume of the reaction suspension.

\subsection{CNF aerogel preparation}

The reaction conditions allowing for complete coverage of the CNFs (above $1 \mathrm{mg} \mathrm{mL}{ }^{-1}$ ) were scaled up 50 times to prepare sufficient material for aerogel preparation (with the only difference of using mechanical stirring at $300 \mathrm{rpm}$ ). The neat $\mathrm{CNF}$ and the coated CNF suspensions were both adjusted to a solid content of $5 \mathrm{mg} \mathrm{mL} \mathrm{mL}^{-1}$ (resulting in a final density of $0.005 \mathrm{~g} \mathrm{~cm}^{-3}$ for the aerogels). The suspensions were subsequently poured into cubic-shaped silicone moulds, which were frozen from the bottom up using liquid nitrogen (only the bottom surface of the mould was in contact with the liquid nitrogen). The moulds were then transferred into a commercial freeze drier (Scanvac Coolsafe) and dried over a period of 3 days (in order to ensure complete evaporation of water inside the nano-sized network).

\subsection{Silica nanotube aerogel preparation}

The prepared $\mathrm{CNF}-\mathrm{SiO}_{2}$ aerogels were converted into silica nanotube aerogels via thermal treatment at $400{ }^{\circ} \mathrm{C}$ for $6 \mathrm{~h}$ in air. A shorter time $(c a .1 \mathrm{~h})$ resulted in only a partial conversion of the material due to the good thermal insulation of the aerogels. The conversion and stability of the final silica nanotube aerogel were demonstrated by placing a cubic $\mathrm{CNF}-\mathrm{SiO}_{2}$ aerogel side-byside with a cube of neat CNF aerogel in a thermal gradient (200$400{ }^{\circ} \mathrm{C}$, top-bottom surface). The cross-sections of the samples were photographed through a transparent window of borosilicate glass every $4 \mathrm{~s}$ for $1 \mathrm{~h}$ (time-lapse video available in the $\mathrm{ESI} \dagger$ ).

\subsection{Characterization}

Transmission electron micrographs were obtained using a Hitachi HT7700 microscope operating at $100 \mathrm{kV}$. Samples of particles were deposited onto holey carbon-coated 400 mesh copper grids (TED PELLA, USA) from ultrasonicated suspensions of particles in ethanol. Scanning electron microscopy was performed on a Hitachi S-4800, after Pt/Pd sputtering. A MettlerToledo SDTA/TGA905 thermo-balance was used to obtain the mass loss of the nanoparticle samples before and after the surface modification. Prior to the measurements, the samples were heated at $140{ }^{\circ} \mathrm{C}$ for $20 \mathrm{~min}$ to eliminate water adsorbed on the CNF surface. In the TG experiments the samples were heated from 100 to $800{ }^{\circ} \mathrm{C}$ at a heating rate of $10{ }^{\circ} \mathrm{C} \mathrm{min}{ }^{-1}$ in $70 \mu \mathrm{L}$ alumina crucibles under both nitrogen and oxygen atmospheres. X-ray diffraction (XRD) measurements were conducted on the compressed aerogel cubes (resulting in thin square-shaped films) using a PANalytical X'Pert Pro diffractometer (Almelo, The Netherlands) fitted with an Empyrean $\mathrm{Cu}$ $\mathrm{K} \alpha$ tube $(45 \mathrm{kV}, 45 \mathrm{~mA})$ and a $1 \mathrm{D}$ X'Celerator detector with a 1.00 arcmin step size.

\section{Results and discussion}

\subsection{Morphology of silica-coated CNFs}

Fig. 1 shows that the preparation of completely coated nanofibrils relied on finding a concentration of the uncoated CNFs in suspension (Fig. 1a) that matched with the number of $\mathrm{SiO}_{2}$ nuclei formed and the full amount of silica condensed to the surface area of the CNFs. $0.27 \times 10^{-3}$ moles of the condensed TEOS precursor $(0.06 \mathrm{~mL})$ were unable to cover the $1.27 \mathrm{~m}^{2} \mathrm{CNF}$ surface area when $8 \mathrm{mg}\left(3 \mathrm{mg} \mathrm{mL}^{-1}\right)$ of CNFs was used from the suspension of extracted CNFs, Fig. $1 \mathrm{~b}$ and e. Complete coverage was reached only when the amount of extracted CNFs had been reduced to $2.7 \mathrm{mg}$ and the targeted CNF surface area was decreased to $0.43 \mathrm{~m}^{2}$, as shown in Fig. $1 \mathrm{c}$ and $\mathrm{f}$. At this point, the effective amount of TEOS per mg of CNFs was 0.1 moles per $g$ of CNFs. The silica shell on the fibres had an average thickness of ca. $20 \mathrm{~nm}$ (measurements of $c a .40$ particles), where the thinnest coated sections were ca. $15 \mathrm{~nm}$ and the thickest $45 \mathrm{~nm}$. The 
thicker sections corresponded to regions where the silica had grown as nucleated half-spheres on previously formed silica (Fig. 1f). The cause for this formation of the uneven necklacelike morphology stemmed from a heterogeneous and timedifferentiated nucleation along the fibres on the most favourable/accessible spots on the CNFs. Fig. 1d-f show greater magnifications on selected areas of the micrographs on the top. In Fig. 1f, the CNFs are visible inside the nano-necklace of silica and cellulose. The whitish lines inside the silica-cellulose hybrid denote the interfacial region between the surface of the CNF and the silica, and are visible due to a lower specific density of the material in this region. Silica nanoparticles have been reported to show a density of $c a .2 \mathrm{~g} \mathrm{~cm}^{-3},{ }^{41}$ whereas CNFs from bacterial cellulose have a density of $1.59 \mathrm{~g} \mathrm{~cm}^{-3}{ }^{42}$ However, the silica particle coatings may show a density as low as $0.9 \mathrm{~g} \mathrm{~cm}^{-3}$, depending on the growth conditions adopted for the condensation of the TEOS precursor. ${ }^{43}$

An observation from multiple micrographs was that the silica phase appeared to embed the extracted CNFs in their separated state, which can be seen when comparing the thicknesses of the non-coated fibrils in Fig. $1 \mathrm{~d}$ and coated fibrils in Fig. 1e and $\mathrm{f}$. The average diameter of the coated fibrils (visible through the silica particles) was $15-20 \mathrm{~nm}$, whereas the CNFs in
Fig. 1d show an average diameter of $30-40 \mathrm{~nm}$. This size difference was related to the strong tendency of the non-coated fibrils to organize in bundles of 2-4 fibrils and also their tendency to become strongly associated with the carbon surface of the grid, making them appear wider (thicker). The arrow in Fig. 1d points to one of these bundles that consists of at least 2 fibrils and shows that the fibrils appear thinner when crossing the vertically positioned bundle of fibrils at the right side of the micrograph as it was lifted from the surface of the grid at this particular point.

\subsection{Reaction conditions for efficient silica coating}

Fig. 2a-c show the importance of selecting the proper reaction medium composition on the overall outcome of the precipitation of silica in the vicinity of the CNFs. In all the experiments, the amounts of CNFs, TEOS and ammonia were kept constant. When methanol was used as the main solvent, a uniform formation of coating on the CNF surfaces did not occur. Only occasionally, small sub-10 nm grains could be observed on the surface of the fibrils and most of the condensed TEOS was present as poorly structured silica gel (sub-5 $\mathrm{nm}$ particles) separated from the CNFs (see Fig. 2a). This sort of a nanostructured silica gel was previously reported in a more


Fig. 1 Transmission electron micrographs of uncoated cellulose nanofibrils with a surface area of ca. $160 \mathrm{~m}^{2} \mathrm{~g}^{-1}$ and an average overall length of 2-3 $\mu \mathrm{m}$ (a), and silica embedded CNFs coated in 2-propanol at different CNF concentrations: $3 \mathrm{mg} \mathrm{mL}^{-1}$ (b) and $1 \mathrm{mg} \mathrm{mL}^{-1}$ (c). (d), (e) and (f) highlight areas in (a), (b) and (c), respectively. The uncoated and coated CNFs were deposited separately from an ethanol solution, and associated together during evaporation of the ethanol. 
concentrated state as a matrix for the preparation of silicacellulose aerogels. ${ }^{44}$ Fig. $2 \mathrm{~b}$ shows the result of using ethanol instead of methanol, which resulted in a significantly increased size of the silica particles and proper condensation onto the fibril surfaces. However, the randomly organized particles along the fibrils and their poor coverage in combination with the observed size difference indicated a very heterogeneous formation of the silica phase in the ethanol-based solution. A further increase in the alkyl chain length of the alcohol (i.e. by changing ethanol to 2-propanol) resulted in a more homogeneous morphology, Fig. 2c. All the fibrils were completely covered as cores in silica shells, which consisted of half-spheres of silica that had grown together by the condensation occurring at the later stages of the reaction.

The effect of the alcohol chain length (namely, the molecular weight) on the size/morphology of the silica particles/network can be mainly attributed to the different relative permittivities $\varepsilon$ (dielectric constant) of the alcohols. With reference to our system, $\varepsilon$ scales inversely with the molecular weight of the alcohol used, equalling 32.6, 24.55, and 18.3 for methanol, ethanol, and 2-propanol, respectively. The use of methanol as a solvent resulted in an increase in the rate of hydrolysis due to the higher polarity and the smallest steric hindrance compared to that of the other solvents, i.e. ethanol and 2-propanol. ${ }^{39,45}$ The high hydrolysis rate for the methanol-based system explained the poorly structured, grainy network formed in the vicinity of the cellulose fibrils. As observed in other TEOS systems, an increase in the hydrolysis rate may also yield branched morphologies of the final silica network rather than spherical particles. ${ }^{46}$ On the other hand, the more sterically hindered longer-chain 2-propanol provided a lower hydrolysis rate, limited the nucleation rate, and promoted growth directly on the cellulose surface. Accordingly, the hydrolysed TEOS $\left(\mathrm{Si}(\mathrm{OH})_{4}\right)$ concentration never exceeded the critical limiting supersaturation concentration, thus only growth onto already formed surfaces occurred..$^{39,47-49}$

It is apparent from the results that the alcohol configuration had the most dominant effect on the condensation chemistry in the presence of the CNFs. It was also observed that the ammonia concentration had an essentially small effect on the formation of silica, only showing a slightly more even distribution for the highest concentration applied, see ESI Fig. S2. $\dagger$ All further characterization was therefore focused on the fibrils coated in the 2-propanol solutions.

\subsection{Thermal properties of silica-coated CNFs}

Fig. $3 \mathrm{a}$ and $\mathrm{b}$ show the thermal behaviour of the silica-coated CNFs as compared to uncoated CNFs in nitrogen and oxygen. The presence of different silica coverages on the CNF surface increased the onset of the degradation temperature with $c a .50$ and $70{ }^{\circ} \mathrm{C}$ in nitrogen (to 315 and $335^{\circ} \mathrm{C}$ ) for the semi-complete ( $3 \mathrm{mg} \mathrm{mL}^{-1}$ ) and the complete $\left(1 \mathrm{mg} \mathrm{mL}^{-1}\right)$ coverage, respectively, from $265{ }^{\circ} \mathrm{C}$ for the uncoated CNF (in nitrogen), Fig. 3a.

The same trend was observed for the samples heated in an oxygen atmosphere but with a smaller increase in the degradation stability, i.e. 25 and $47{ }^{\circ} \mathrm{C}$ from $258{ }^{\circ} \mathrm{C}$ (semi- and
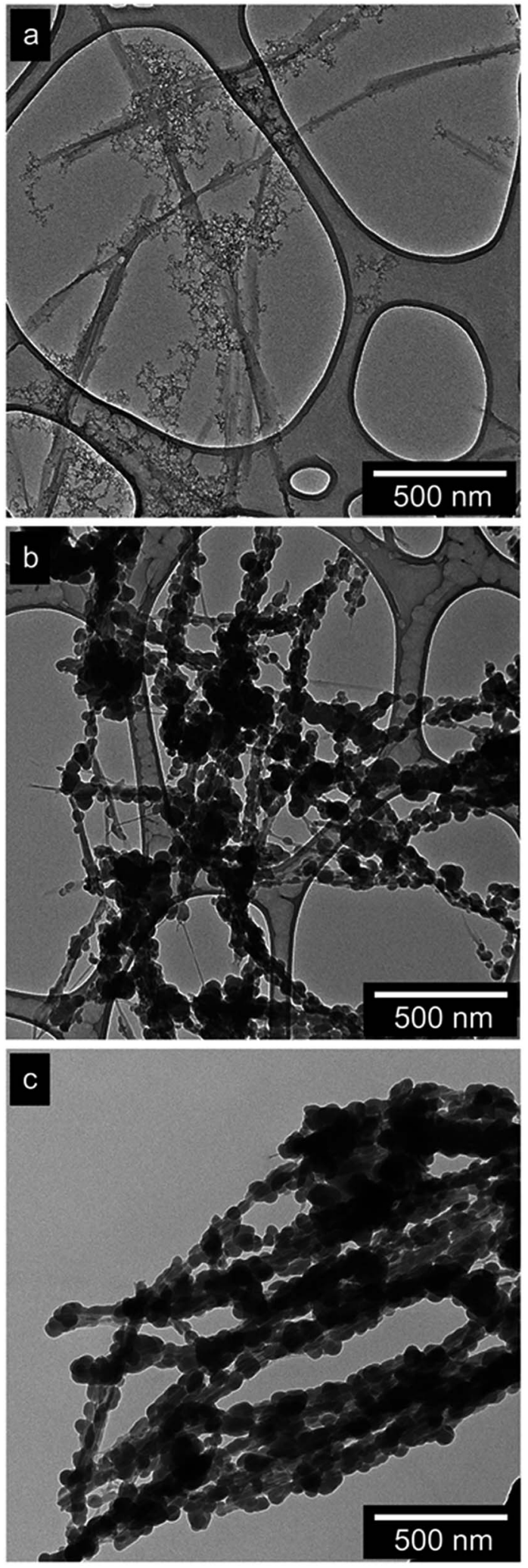

Fig. 2 Micrographs showing the effect of the reaction medium on the precipitation of the same concentration of silica in the vicinity of CNFs. Reaction medium: (a) methanol; (b) ethanol; (c) 2-propanol. The amount of water was $25 \mathrm{wt} \%$ in all cases. 

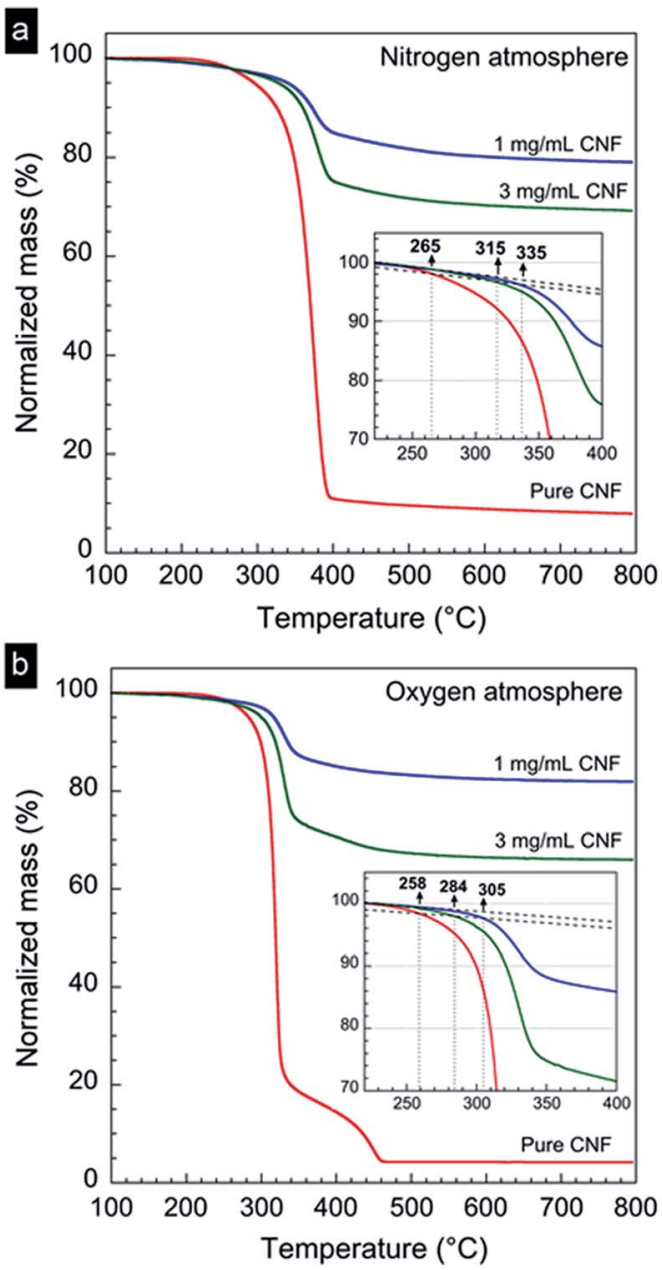

Fig. 3 TGA curves of silica-coated CNFs, (a) under a nitrogen atmosphere, (b) under an oxygen atmosphere. All samples were run thrice to ensure accuracy in the plotted data. 1 and $3 \mathrm{mg} \mathrm{mL}^{-1}$ refer to the concentrations of CNFs in the reactions.

complete coverage), Fig. 3b. Table 1 presents a summary of previously highest reported temperatures of the onset and maximum mass loss rate for cellulose nanofibrils, together with data for the silica shell-covered CNFs. The temperature for the onset of the degradation is defined as the temperature at which the materials had lost $1 \mathrm{wt} \%$ mass as determined from a fitted straight line drawn from 150 to $200{ }^{\circ} \mathrm{C}$ for each TGA sample (to eliminate possible strongly-bound crystalline water). ${ }^{\mathbf{1 4}}$

The complete silica coverage of the CNFs also resulted in an increase in the maximum mass loss-rate temperature to $c a$. $377{ }^{\circ} \mathrm{C}$ from $374{ }^{\circ} \mathrm{C}$ (under a nitrogen atmosphere), and to $\mathrm{ca}$. $331{ }^{\circ} \mathrm{C}$ from $320{ }^{\circ} \mathrm{C}$ (under an oxygen atmosphere). The small difference in the maximum mass loss rate temperatures compared to that of the onset of the degradation temperatures indicated that the silica coating on the CNFs was not able to prevent the bulk degradation once it started, even for the thickest coatings. The values for the maximum mass loss rate temperatures were therefore regarded as less useful for comparisons of degradation stability, and from an engineering point of view the maximum temperature the material can withstand is better represented by the onset of the material degradation.
The residual $\mathrm{SiO}_{2}$ mass for the samples allowed for calculating the coating reaction efficiency and $0.06 \mathrm{~mL}(0.27 \mathrm{mmol})$ of the used TEOS should convert into $16.1 \mathrm{mg}$ of $\mathrm{SiO}_{2}$ assuming $100 \%$ efficiency. Considering the total weight of cellulose (2.7 $\mathrm{mg}$ and $8 \mathrm{mg}$ ), the reactions should therefore result in a $\mathrm{SiO}_{2}$ content of 85.7 and $66.8 \mathrm{wt} \%$ of the hybrid fibres. The mass loss (Fig. 3b) showed that the final $\mathrm{SiO}_{2}$ content (deducting the mass of residual ash) after ultra-sonication and washing was 80 and $65 \mathrm{wt} \%$ for the two concentrations of coated fibres. This corresponded to yields for the coating procedure of 93 and $97 \%$ for the 1 and $3 \mathrm{mg} \mathrm{mL}{ }^{-1}$ systems, confirming that the silica condensed in situ on the surface of the fibres. Hence, only a small amount of silica was lost in the entire processing (including the ultra sonication and washing of the fibres).

An unexpected observation from the measurements can be seen in Fig. 3b, which shows that the degradation of the uncoated fibrils (oxygen) slowed down considerably as $80 \mathrm{wt} \%$ of the material was degraded at $320{ }^{\circ} \mathrm{C}$, and complete degradation was not reached until at a temperature of $c a .460{ }^{\circ} \mathrm{C}$. It is suggested that this transition was due to the extensive formation of char at the surface of the sample, which restricted the accessibility of oxygen. ${ }^{\mathbf{1 3}, 50}$ This phenomenon was not observed for the identical fibrils degrading under a nitrogen atmosphere at ca. $50{ }^{\circ} \mathrm{C}$ higher maximum mass loss rate temperature, i.e. $374{ }^{\circ} \mathrm{C}$ (Fig. 3a). Instead, the degradation pattern showed a more continuous weight-loss, which may have included both degradation mechanisms visible as separated mass-loss occasions in Fig. $3 \mathrm{~b}$. The residual left in the crucibles after the heat treatment in a nitrogen atmosphere was ca. 8 wt\% black char, whereas the oxygen conditions resulted in $c a$. $4 \mathrm{wt} \%$ white char. The black char was therefore interpreted as a sign of incomplete transformation of the carbon material into carbon dioxide. It is suggested that the presence of an extensive amount of 'capping' char, in combination with very low (ppm) amounts of oxygen in the nitrogen gas, was the explanation to the continuous weight loss up to $800{ }^{\circ} \mathrm{C}$. This behaviour indicated that the major role of the silica phase was similar to that of the 'capping' char in that it prevented the diffusion of oxygen into the cellulose fibrils, and limited the outgassing of degradation products, thereby delaying the degradation of the cellulose.

\subsection{Silica and char residuals post high-temperature treatment}

The silica-cellulose fibril residuals collected from the TGA crucible after the high-temperature treatment in oxygen are shown in Fig. 4. The cellulose had been completely removed (see XRD, ESI Fig. S3†), and all the internal structural support from the CNFs was eliminated. Fig. 4c shows that the hybrid silica/cellulose fibrils had been converted into hollow silica nanotubes. The results also confirmed that the silica coatings before the heat treatment completely covered the CNFs. The silica nanotubes seldom appeared broken at their ends where poor silica fibril coverage had been the case, i.e. leaving open tube morphologies (see Fig. 4c). The most frequent endings were spherical shaped particles that had nucleated at the end points of the removed fibrils (see Fig. 4b). The brighter interior 
Table 1 Data from previous articles were obtained by digital reading of scanned plotted values in graphs. Definitions of temperature zones were according to the main text

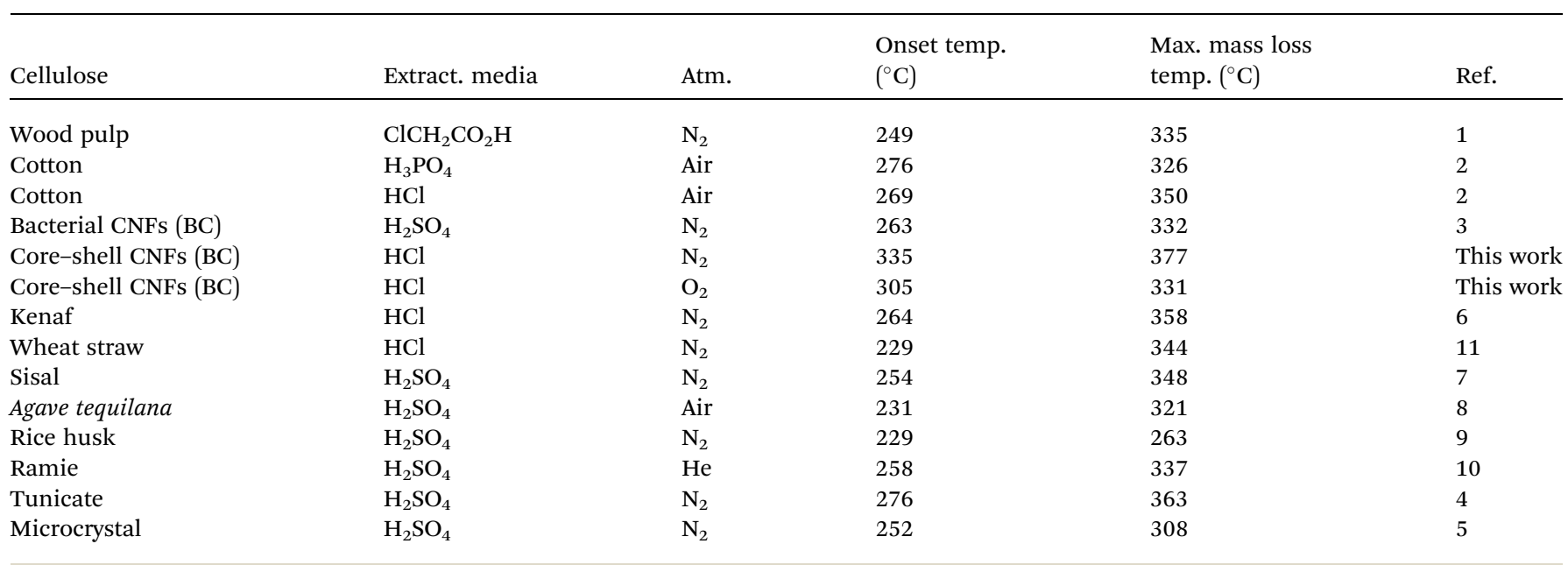

of the nanotube in Fig. $4 \mathrm{c}$ also shows that the CNFs inside the coated hybrid fibres were predominantly present as bundles prior to their removal. The $c a .30 \mathrm{~nm}$ width of the removed fibril bundle was consistent with a coating deposited on 2-3 CNFs, as discussed in Section 3.1.

\subsection{Hybrid fibre aerogels and their high-temperature behaviour}

Fig. 5 shows that the hybrid silica/cellulose fibres could be formed into aerogels by simple freeze-drying, Fig. 5a (right image). The macroscopic appearance of the hybrid foam was similar to the pure cellulose foam (Fig. 5a, left image) whereas its mechanical integrity was higher (easier to handle) and more shape-precise to the mould used when freeze-drying the cubes. The densities of the solid cubes were $0.005 \mathrm{~g} \mathrm{~cm}^{-3}$ in both cases based on the weight and volume of the cubes. The photographs in $b$ and $c$ show the result of the cellulose phase degradation when the foams were heated from room temperature to $400{ }^{\circ} \mathrm{C}$ (heating rate: $20^{\circ} \mathrm{C} \mathrm{min}^{-1}$ ). Whereas the pure cellulose aerogel lost more than $99.9 \%$ of its volume, the hybrid foam retained its shape even when the embedded cellulose had been completely degraded. The intact shape of the hybrid foam was due to the presence of the remaining uniform and intact network of the silica nanotubes.

Fig. $5 \mathrm{~d}-\mathrm{f}$ show the wall structures of the foams before and after the heating experiment. In both cases, the macroscopic organisation of the walls was dictated by the ice crystals formed during the quenching of the fibre suspensions in a manner similar to that reported in the preparation of cellulose-based biofoams. ${ }^{5,51}$ The lower images show that the in situ condensed silica particles, which entirely cover the cellulose nanofibrils, prevented their inter-condensation into sheet-like flats visible in Fig. 5d, compare Fig. 5e for the hybrid material. From Fig. $5 f$ it is clear that even in the absence of the fibrillar intercondensation the aerogel structure remained after removal of the cellulose phase. Cellulose silica composites have previously been demonstrated by a few research groups. ${ }^{52-54}$ So far, however, the fabrication of these from fully embedded individual cellulose fibrils that can be used as building blocks for
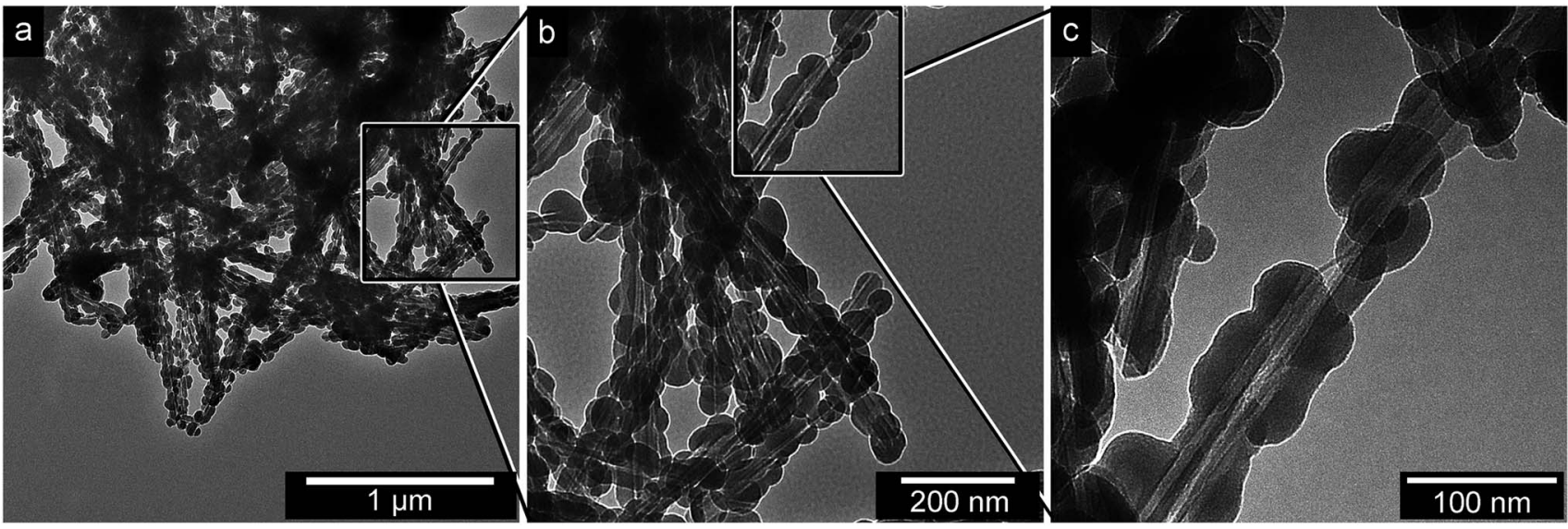

Fig. 4 Transmission electron micrographs of the silica remains after oxygen degradation of the cellulose nanofibril interior (a-c). 

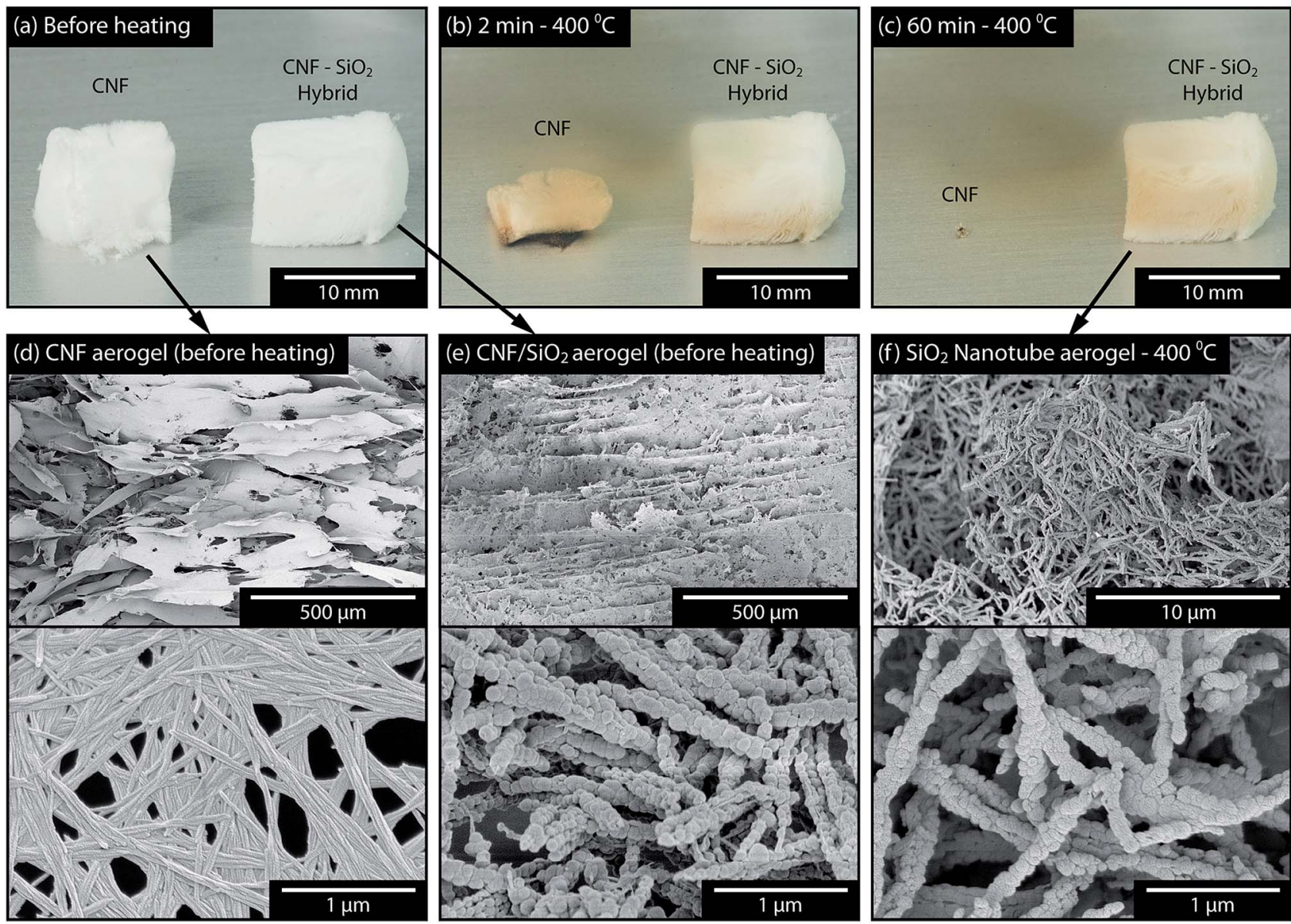

Fig. 5 Photographs with corresponding scanning electron micrographs of the cellulose, hybrid silica/cellulose and silica nanotube aerogels at different stages during transformation. The lower micrographs of Fig. $5 \mathrm{~d}-\mathrm{f}$ are larger magnifications of the wall morphologies in the above micrographs.

silica nanotube aerogels or as more thermally stable fibrils for liquid processing has not been reported. A time-lapse video on the thermal degradation and collapse of the cellulose aerogel compared with the silica hybrid aerogel is included in the ESI (video $1 \dagger$ ), showing that the hybrid material retains its shape even after conversion into silica nanotubes at $400{ }^{\circ} \mathrm{C}$.

\section{Conclusions}

A key parameter for growth of solid silica layers on the surface of cellulose nanofibrils is to use a reaction medium that permits in situ nucleation on the surface of the fibrils, i.e. by avoiding premature silica nucleation in the reaction medium. An appropriate amount of the silica precursor for proper nucleation and growth on the surface of the CNFs is required to come in contact with the reactive surface of the CNFs (which can be established by dilution experiments). The results show that under these premises it is possible to realize an average efficiency of the entire coating procedure of $c a .95 \%$ yield (after the coating reaction combined with ultra-sonication and washing). The coated fibrils were present as individual fibrils, or bundles of 2-3 CNFs that remained from a limited separation during the extraction of the CNFs. A full silica coverage on the CNFs limited the accessibility of oxygen and restricted degassing of degradation products, resulting in delayed CNF degradation. The shifts in the onset mass loss temperature to higher temperatures were ca. $70{ }^{\circ} \mathrm{C}$ for $\mathrm{N}_{2}$ and $50{ }^{\circ} \mathrm{C}$ for $\mathrm{O}_{2}$. Complete CNF degradation/ pyrolysis allowed for the preparation of hollow silica nanotubes.

It is also demonstrated that the core-shell structured CNFsilica hybrid fibres allowed for simple freeze-drying to be used to prepare mouldable silica cellulose aerogels. Here, the slender CNF network penetrating the aerogel served as a structural support, which prevented the collapse of the aerogel during drying. The CNF-silica hybrid aerogels could further be converted into silica nanotube aerogels by simple heating to $400^{\circ} \mathrm{C}$ (video available in the ESI $\dagger$ ). The preparation scheme may open a new avenue for large-scale fabrication of silica aerogels for thermal insulation, which has been traditionally relying on super-critical drying methods.

\section{Acknowledgements}

The Wallenberg Wood Science Centre (WWSC) is acknowledged, as well as Lars-Erik Thunholms stiftelse and the China Scholarship Council. 


\section{Notes and references}

1 T. Zimmermann, E. Pöhler and T. Geiger, Adv. Eng. Mater., 2004, 6, 754-761.

2 H. Fukuzumi, T. Saito, T. Iwata, Y. Kumamoto and A. Isogai, Biomacromolecules, 2009, 10, 162-165.

3 F. W. Brodin, Ø. W. Gregersen and K. Syverud, Nord. Pulp Pap. Res. J., 2014, 29, 156-166.

4 T. O. J. Blomfeldt, R. T. Olsson, M. Menon, D. Plackett, E. Johansson and M. S. Hedenqvist, Macromol. Mater. Eng., 2010, 295, 796-801.

5 Q. Wu, R. L. Andersson, T. Holgate, E. Johansson, U. W. Gedde, R. T. Olsson and M. S. Hedenqvist, J. Mater. Chem. A, 2014, 2, 20996-21009.

6 A. J. Svagan, M. A. S. A. Samir and L. A. Berglund, Adv. Mater., 2008, 20, 1263-1269.

7 A. Broido, in Thermal Uses and Properties of Carbohydrates and Lignins, ed. F. S. V. S. A. Tillman, Academic Press, 1976, pp. 19-36, DOI: 10.1016/B978-0-12-637750-7.50006-6.

8 F. Shafizadeh, R. H. Furneaux, T. G. Cochran, J. P. Scholl and Y. Sakai, J. Appl. Polym. Sci., 1979, 23, 3525-3539.

9 M. J. Antal Jr and G. Varhegyi, Ind. Eng. Chem. Res., 1995, 34, 703-717.

10 J. B. Wooten, J. I. Seeman and M. R. Hajaligol, Energy Fuels, 2004, 18, 1-15.

11 J. Cai, S. Kimura, M. Wada and S. Kuga, Biomacromolecules, 2009, 10, 87-94.

12 J. Zeng, S. Liu, J. Cai and L. Zhang, J. Phys. Chem. C, 2010, 114, 7806-7811.

13 J. Alongi, M. Ciobanu and G. Malucelli, Carbohydr. Polym., 2012, 87, 2093-2099.

14 M. Roman and W. T. Winter, Biomacromolecules, 2004, 5, 1671-1677.

15 M. Martínez-Sanz, A. Lopez-Rubio and J. M. Lagaron, Carbohydr. Polym., 2011, 85, 228-236.

16 G. Tesoro, S. Sello and J. Willard, Text. Res. J., 1969, 39, 180190.

17 G. Rosace, V. Migani, E. Guido and C. Colleoni, Flame Retardant Finishing for Textiles in Flame Retardants: Polymer Blends, Composites and Nanocomposites, ed. P. M Visakh and Y. Arao, Springer, 2015, pp. 209-246.

18 A. R. Horrocks, B. K. Kandola, P. J. Davies, S. Zhang and S. A. Padbury, Polym. Degrad. Stab., 2005, 88, 3-12.

19 J. E. Hendrix, G. L. Drake and R. H. Barker, J. Appl. Polym. Sci., 1972, 16, 257-274.

20 B. F. Gilliland and B. F. Smith, J. Appl. Polym. Sci., 1972, 16, 1801-1816.

21 O. A. Battista, Ind. Eng. Chem., 1950, 42, 502-507.

22 S. Camarero Espinosa, T. Kuhnt, E. J. Foster and C. Weder, Biomacromolecules, 2013, 14, 1223-1230.

23 A. Dufresne, Nanocellulose: from nature to high performance tailored materials, Walter de Gruyter, 2012.

24 F. Fahma, S. Iwamoto, N. Hori, T. Iwata and A. Takemura, Cellulose, 2010, 17, 977-985.

25 L. Petersson, I. Kvien and K. Oksman, Compos. Sci. Technol., 2007, 67, 2535-2544.
26 N. Johar, I. Ahmad and A. Dufresne, Ind. Crops Prod., 2012, 37, 93-99.

27 I. A. Sacui, R. C. Nieuwendaal, D. J. Burnett, S. J. Stranick, M. Jorfi, C. Weder, E. J. Foster, R. T. Olsson and J. W. Gilman, ACS Appl. Mater. Interfaces, 2014, 6, 6127-6138. 28 B. He, S. J. Son and S. B. Lee, Langmuir, 2006, 22, 8263-8265. 29 T.-W. Lin and H.-H. Shen, Nanotechnology, 2010, 21, 365604. 30 Y. Yu, H. Qiu, X. Wu, H. Li, Y. Li, Y. Sakamoto, Y. Inoue, K. Sakamoto, O. Terasaki and S. Che, Adv. Funct. Mater., 2008, 18, 541-550.

31 L. Wang, S. Tomura, F. Ohashi, M. Maeda, M. Suzuki and K. Inukai, J. Mater. Chem., 2001, 11, 1465-1468.

32 M. Zhang, W. Zhang and S. Wang, J. Phys. Chem. C, 2010, 114, 15640-15644.

33 M. Yamanaka, Y. Miyake, S. Akita and K. Nakano, Chem. Mater., 2008, 20, 2072-2074.

34 J. H. Jung, S. Shinkai and T. Shimizu, Nano Lett., 2002, 2, 1720.

35 Y. Zhang, X. Liu and J. Huang, ACS Appl. Mater. Interfaces, 2011, 3, 3272-3275.

36 X. Yang, H. Tang, K. Cao, H. Song, W. Sheng and Q. Wu, J. Mater. Chem., 2011, 21, 6122-6135.

37 R. T. Olsson, R. Kraemer, A. López-Rubio, S. Torres-Giner, M. J. Ocio and J. M. Lagarón, Macromolecules, 2010, 43, 4201-4209.

38 R. T. Olsson, M. A. S. Azizi Samir, G. Salazar Alvarez, L. Belova, V. Strom, L. A. Berglund, O. Ikkala, J. Nogues and U. W. Gedde, Nat. Nanotechnol., 2010, 5, 584-588.

39 M. T. Harris, R. R. Brunson and C. H. Byers, J. Non-Cryst. Solids, 1990, 121, 397-403.

40 W. Stöber, A. Fink and E. Bohn, J. Colloid Interface Sci., 1968, 26, 62-69.

41 A. van Blaaderen and A. Vrij, J. Colloid Interface Sci., 1993, 156, 1-18.

42 J. Sugiyama, R. Vuong and H. Chanzy, Macromolecules, 1991, 24, 4168-4175.

43 D. Liu, A. M. Pourrahimi, L. K. H. Pallon, R. L. Andersson, M. S. Hedenqvist, U. W. Gedde and R. T. Olsson, RSC Adv., 2015, 5, 48094-48103.

44 H. Sai, L. Xing, J. Xiang, L. Cui, J. Jiao, C. Zhao, Z. Li and F. Li, J. Mater. Chem. A, 2013, 1, 7963-7970.

45 A. Venkateswara Rao, G. M. Pajonk and N. N. Parvathy, J. Mater. Sci., 1994, 29, 1807-1817.

46 J. Retuert, R. Quijada, V. Arias and M. Yazdani-Pedram, J. Mater. Res., 2003, 18, 487-494.

47 S. Sadasivan, A. Dubey, Y. Li and D. Rasmussen, J. Sol-Gel Sci. Technol., 1998, 12, 5-14.

48 J. S. Park, H. J. Hah, S. M. Koo and Y. S. Lee, J. Ceram. Process. Res., 2006, 7, 83.

49 V. K. LaMer and R. H. Dinegar, J. Am. Chem. Soc., 1950, 72, 4847-4854.

50 G. Brancatelli, C. Colleoni, M. R. Massafra and G. Rosace, Polym. Degrad. Stab., 2011, 96, 483-490.

51 A. J. Svagan, M. A. Samir and L. A. Berglund, Adv. Mater., 2008, 20, 1263-1269.

52 J. Cai, S. Liu, J. Feng, S. Kimura, M. Wada, S. Kuga and L. Zhang, Angew. Chem., 2012, 124, 2118-2121. 
53 F. Liebner, E. Haimer, M. Wendland, M.-A. Neouze, K. Schlufter, P. Miethe, T. Heinze, A. Potthast and T. Rosenau, Macromol. Biosci., 2010, 10, 349-352.
54 S. Zhao, Z. Zhang, G. Sèbe, R. Wu, R. V. Rivera Virtudazo, P. Tingaut and M. M. Koebel, Adv. Funct. Mater., 2015, 25, 2326-2334. 